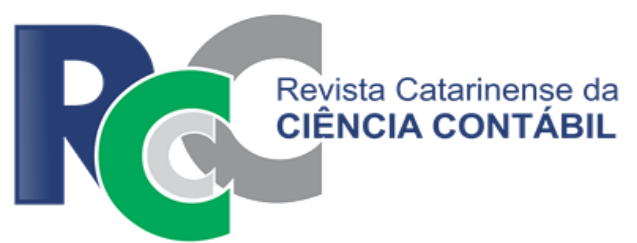

\title{
FATORES DE SOCIALIZAÇÃO ANTECIPATÓRIA: UM ESTUDO COM ALUNOS DE CIÊNCIAS CONTÁBEIS
}

\section{FACTORS OF ANTECIPATORY SOCIALIZATION: A STUDY WITH STUDENTS OF ACCOUNTING SCIENCES}

\author{
EDENISE APARECIDA DOS ANJOS \\ Universidade Federal de Santa Catarina. Endereço: Universidade \\ Federal de Santa Catarina, Campus Universitário Trindade / Centro \\ Socioeconômico / Departamento de Ciências Contábeis / \\ Florianópolis/SC / Brasil. \\ D http://orcid.org/0000-0002-6247-5293 \\ E-mail: edeniseanjos@outlook.com
}

\section{EDICREIA ANDRADE DOS SANTOS}

Universidade Federal de Santa Catarina. Endereço: Universidade Federal de Santa Catarina, Campus Universitário Trindade / Centro Socioeconômico / Departamento de Ciências Contábeis / Florianópolis/SC / Brasil.

(D)ttp://orcid.org/0000-0001-8745-3579

E-mail: edicreiaandrade@yahoo.com.br

\section{IVANILDO VIANA MOURA}

Universidade Federal do Paraná. Endereço: Universidade Federal do Paraná, Campus Botânico, Departamento de Ciências Contábeis / Av. Prefeito Lothário Meissner, 632 | Curitiba/PR / Brasil.

(1) http://orcid.org/0000-0002-3284-449X

E-mail: ivm.bh.mg@gmail.com

\section{LAURO BRITO DE ALMEIDA}

Universidade Federal do Paraná. Endereço: Universidade Federal do Paraná, Campus Botânico, Departamento de Ciências Contábeis / Av. Prefeito Lothário Meissner, 632 / Curitiba/PR / Brasil.

(1) http://orcid.org/0000-0002-4374-6705

E-mail: gbrito@uol.com.br

\section{ROGÉRIO JOÃO LUNKES}

Universidade Federal de Santa Catarina. Endereço: Universidade Federal de Santa Catarina, Campus Universitário Trindade / Centro Socioeconômico / Departamento de Ciências Contábeis / Florianópolis/SC / Brasil.

(1) http://orcid.org/0000-0003-4232-5746

E-mail: rogeriolunkes@hotmail.com

\section{RESUMO}

Motivação, percepção do ambiente, envolvimento e compromisso profissional caracterizam-se como elementos sociáveis de um indivíduo. Nesse contexto, este estudo investiga os fatores de socialização antecipatória de estudantes universitários de Contabilidade de duas universidades federais do estado do Paraná, sob a sustentação conceitual e metodológica do modelo de Astin. Os dados foram coletados por meio de um questionário adaptado do estudo 
de Ahmad, Anantharaman e Ismail (2012), aplicado a 71 alunos concluintes da graduação no ano de 2016, e foram tratados com as técnicas estatísticas de análise descritiva e de Modelagem de Equações Estruturais via Partial Least Square (PLS). Os achados da pesquisa sugerem que os fatores de motivação e de percepção de ambiente não impactam de forma estatisticamente significativa no envolvimento e, por consequência, no compromisso profissional. Contudo, confirmou-se que a motivação afeta de forma positiva e significativa o comprometimento profissional. Com isso, os resultados instigam pesquisas futuras para incluir outras características cognitivas dos alunos, as quais possam influenciá-los frente ao compromisso profissional.

Palavras-chave: Motivação. Percepção de Ambiente. Envolvimento. Compromisso Profissional.

\begin{abstract}
Motivation, environmental perception, involvement and professional commitment are characterized as sociable elements of an individual. In this context, this study investigates the factors of anticipatory socialization of university students of accounting from two federal universities in the state of Paraná under the conceptual and methodological support of the Astin model. The data were collected through a questionnaire adapted from the study of Ahmad, Anantharaman and Ismail (2012), applied to 71 graduating students in the year 2016, and were treated with the statistical techniques of descriptive analysis and Structural Equation Modeling via Partial Least Square (PLS). The research findings suggest that the motivation and environmental perception factors do not affect in a statistically significant way on the involvement and, consequently, the professional commitment. However, it was confirmed that the motivation positively and significantly affects the professional commitment. Thus, these results instigate future research to include other cognitive characteristics of students that may influence them in the face of professional commitment.
\end{abstract}

Keywords: Motivation. Perception of Environment. Involvement. Professional Commitment.

\title{
1 INTRODUÇÃO
}

A integridade da profissão contábil e a credibilidade das informações financeiras fornecidas pelas empresas têm sido prejudicadas por escândalos, os quais derivam em grande parte das práticas eticamente questionáveis de executivos e profissionais da Contabilidade (Low, Davey \& Hooper, 2008). Para Everett e Tremblay (2014), a divulgação desses escândalos corporativos instiga acreditar que os contadores têm perdido de vista sua orientação moral, comprometendo o prestígio da profissão.

O resultado do trabalho dos profissionais contábeis é de extrema importância para diversos tipos de usuários, como acionistas, credores, empregados, fornecedores, governo, além da própria profissão e da sociedade em geral; e por isso devem atentar-se a sua responsabilidade profissional (Mahdavikhou \& Khotanlou, 2012). Para Borges e Medeiros (2007), os profissionais de Contabilidade devem possuir elementos capazes de colocá-los numa posição diferenciada em relação aos seus pares, por meio da sua capacidade técnica, postura ética e compromisso profissional e social.

O compromisso do profissional, de modo geral, é um elemento crucial no contexto organizacional, cujo êxito no alcance das metas depende em grande parte das estratégias criadas pela organização para envolver o trabalhador, assegurando o compartilhamento de valores, de objetivos e de políticas que garantam trocas equânimes entre as partes (Bastos \& Andrade, 2002). Diante disso, o compromisso profissional do contador torna-se algo imprescindível de ser estudado, haja vista não se saber até que ponto o indivíduo está de fato comprometido com os deveres da profissão e quais são os fatores que influenciam suas atitudes.

As pesquisas sobre comprometimento, apesar de amplamente investigadas no campo organizacional (Borges \& Medeiros, 2007), ainda carecem de mais estudos em outros 
contextos. Investigar, entender e compreender as raízes do compromisso profissional são de grande importância, em especial para identificar os fatores que possam necessitar de intervenção durante o processo de formação de um indivíduo. Barros (2011) argumenta que a escola é um dos agentes mais importantes da socialização antecipatória dos indivíduos. Assume um papel determinante na promoção de atitudes e comportamentos, fundamentais a uma futura adaptação no mundo profissional. Nessa linha, para Ahmad et al. (2012), a existência do compromisso profissional resulta da socialização específica. Acontece no nível do ensino de graduação ou fase antecipatória. Assim, de acordo com os autores, antes do indivíduo ingressar no mercado de trabalho ocorre a aquisição de valores, as atitudes são moldadas e as capacidades cognitivas são desenvolvidas.

Nas instituições de ensino superior (IES), pouca atenção é dada para a aquisição de valores, comportamentos e atitudes necessárias para o aluno assumir o seu papel profissional (Shinyashiki, Mendes, Trevizan, \& Day, 2006). A socialização profissional começa no contexto acadêmico. Estende-se posteriormente para o ambiente de trabalho. Diante disso, Baccaro (2007) pontua que o desenvolvimento dos alunos deve ser o foco das IES, considerando a internalização das normas e dos valores da profissão, pois, quando ingressam no mercado de trabalho, a maneira como foram socializados será um diferencial.

Diante desse contexto, é propósito deste estudo contribuir para a melhoria da compreensão do processo de formação do compromisso profissional que ocorre na fase de socialização antecipatória, desenvolvida no decorrer dos cursos de graduação para bacharel em Ciências Contábeis. Portanto, a questão norteadora dessa investigação é: quais fatores, durante o curso de graduação em Contabilidade, influenciam a construção do compromisso profissional dos alunos com a profissão contábil?

Nessa perspectiva, esta pesquisa segue a mesma linha do estudo de Ahmad et al. (2012), que adequou o modelo de Entrada-Ambiente-Saída (EAR) de Astin (1993), originalmente desenvolvido com a finalidade de identificar fatores de influência significativa do compromisso profissional. Busca-se contribuir, com este estudo, de forma relevante para a formação de uma base de literatura mais robusta sobre as experiências vividas pelos estudantes no período de graduação que tendem a influenciar a formação do compromisso profissional, haja vista que no Brasil pesquisas desse tema, com foco na área de ensino e prática profissional, são ainda incipientes. Outro importante fator é a contribuição para com as IES, uma vez que pode fornecer subsídios de avaliação quanto aos itens que podem ser considerados como mais importantes no processo de formação dos acadêmicos. A pesquisa aponta os fatores que mais contribuem para a formação do compromisso profissional dos estudantes de Ciências Contábeis.

\section{REFERENCIAL TEÓRICO E HIPÓTESES DE PESQUISA}

Neste tópico, serão abordados estudos sobre o compromisso profissional na Contabilidade, a definição e os conceitos de socialização antecipatória dos estudantes de Contabilidade, bem como a teoria de Astin e as hipóteses de pesquisa.

\subsection{Compromisso profissional na Contabilidade}

O compromisso profissional, de acordo com Setyadi (2008), relaciona a natureza do indivíduo com a sua profissão, e inclui a crença, a aceitação dos objetivos e valores profissionais. Nessa linha, na área de Contabilidade, o compromisso profissional é significativo para os profissionais, na medida em que leva a uma maior sensibilidade para as questões de ética e aumenta o envolvimento do trabalho. Ele determina não somente a fidelidade do profissional para com o exercício da profissão, mas também a adesão a códigos de conduta profissionais (Ahmad et al., 2012). A importância desses fatores também é ressaltada por Mahdavikhou e Khotanlou (2012), os quais afirmam que a existência de normas e regras por si só não garantem informações verdadeiras. Os profissionais sem responsabilidades e valores morais podem fornecer relatórios financeiros manipulados.

A questão do compromisso do profissional em relação à profissão contábil tem sido consideravelmente abordada nos últimos anos, devido às crises de confiança causadas por 
muitas falhas éticas (Elias, 2007). A integridade da profissão contábil e a credibilidade das informações financeiras fornecidas pelas empresas têm sido prejudicadas por escândalos, os quais derivam em grande parte das práticas éticas questionáveis de executivos e dos profissionais da Contabilidade (Low, Davey \& Hooper, 2008). Para Everett e Tremblay (2014), a divulgação desses escândalos corporativos instiga a acreditar que os profissionais têm perdido de vista sua orientação moral.

Nessa perspectiva, a falta de moralidade nas práticas contábeis é um fator que pode trazer consequências inestimáveis, pois a Contabilidade trabalha com o patrimônio das empresas. Ademais, o resultado do trabalho dos profissionais contábeis é de extrema importância para diversos tipos de usuários, como acionistas, credores, empregados, fornecedores, governo, além da própria profissão e da sociedade em geral; e por isso devem atentar-se a sua responsabilidade profissional (Mahdavikhou \& Khotanlou, 2012). Assim, esses profissionais devem possuir elementos capazes de colocá-los numa posição diferenciada em relação aos seus pares. Devem utilizar-se de sua capacidade técnica, sua postura ética e seu compromisso profissional e social (Borges \& Medeiros, 2007).

A importância do comportamento ético e da integridade pessoal de um indivíduo, para a profissão e para a nação, deve ser enfatizada na educação dos acadêmicos e também dos profissionais de Contabilidade já formados (Smith, 2003). Para Mastracchio (2005), a educação em Contabilidade deve ensinar métodos para medir as consequências das decisões tomadas pelos contadores. No mesmo sentido, Elias (2007) aponta que a educação para o compromisso profissional na Contabilidade deve começar na sala de aula, ao enfatizar a importância dos relatórios financeiros para os usuários, como também da própria profissão. Assim, observa-se que esses e outros comportamentos devem ser direcionados para um maior compromisso profissional com a área e enfatizados em todas as etapas de um profissional, em especial na de socialização com a profissão.

\subsection{Socialização antecipatória dos estudantes de Contabilidade}

A socialização profissional pode ser entendida como o processo por meio do qual o estudante adquire um sentimento de identidade profissional e desenvolve conhecimentos, habilidades e comprometimento com a profissão (Waugaman \& Lohrer, 2000). Esse processo de socialização ocorre de acordo com acontecimentos, encontros, ou de necessidades e desejos dos indivíduos. Podem ser incluídos nesse contexto os valores dos grupos com os quais se relaciona. Nesse sentido, Dubar (2005) argumenta que a socialização não é somente a transmissão de valores, regras e normas, mas também faz com que o indivíduo desenvolva uma representação de um determinado ambiente ou área de especialização.

O processo de socialização profissional é desenvolvido em três fases, conforme Ardts, Janse e Van Der Velde (2001): (i) a fase antecipatória, que ocorre durante o ensino superior; (ii) fase do primeiro encaminhamento para o exercício das atividades profissionais e (iii) fase da aquisição de experiência após vários anos de trabalho profissional. Acerca da primeira fase, a de socialização antecipatória, Pitney (2002) a define como o processo ou estágio pelo qual os indivíduos aprendem a internalizar traços profissionais únicos para a profissão escolhida. $\mathrm{Na}$ profissão contábil, foco deste estudo, o processo de socialização ocorre na fase antecipatória. É um processo contínuo, que ocorre durante o período do curso superior em Ciências Contábeis.

A formação dos graduandos é um processo complexo. É quando os indivíduos passam do nível de estudantes para o de profissionais. Nesse sentido, Baccaro e Shinyashiki (2011) discorrem que eles são preparados em um nível educacional avançado para assumir responsabilidades pela prática de uma carreira caracterizada por um alto nível de autonomia, dentro do escopo de uma especialidade intelectual particular. As expectativas acerca da prática profissional conduzem ao ensino normativo, com valores, ética, comprometimento pessoal e profissional. Inclinam os indivíduos para uma orientação de serviços e desenvolvimento de conhecimentos e habilidades (Weidman, Twale \& Stein, 2001).

A socialização antecipatória, mesmo com origem durante o curso superior, se estende a toda a vida profissional do indivíduo. Portanto, as IES devem focar no desenvolvimento de seus alunos e considerar a internalização das normas e dos valores da carreira, pois quando estes 
ingressam no mercado de trabalho, a maneira como foram socializados será um diferencial (Baccaro, 2007). Nessa linha, para Ahmad et al. (2012), as IES devem garantir que o ambiente de ensino seja propício para atividades de aprendizagem e envolvimento do aluno, incentivando-o e iniciando-o corretamente na carreira profissional.

No contexto contábil brasileiro, as pesquisas sob socialização antecipatória são incipientes. Contudo, no contexto internacional, diversos estudos apontam efeitos positivos sobre a socialização antecipatória dos estudantes de Contabilidade em assuntos diversos, como ambiente, ética e características dos estudantes (Elias, 2006, 2007, 2008; Saat, Porter \& Woodbine, 2012; Farag, 2016). Ahmad et al. (2012) argumentaram que níveis mais elevados de socialização antecipatória (compreendida em motivação, percepção do ambiente e envolvimento dos estudantes de Contabilidade) resultaram em níveis mais elevados de compromisso profissional.

\subsection{Modelo de Astin}

Este estudo investiga quais fatores, no curso de graduação em Contabilidade, influenciam a construção do compromisso profissional. Está alicerçado na teoria do envolvimento do aluno e no modelo de Astin, estruturado com base em três elementos fundamentais: (i) entrada, (ii) ambiente e (iii) saída dos estudantes de ensino superior.

As experiências vividas pelos estudantes no período de graduação são explicadas pelos modelos de impacto da universidade. Para Pascarella e Terenzini (2005), tais modelos atribuem as mudanças cognitivas e afetivas que ocorrem no aluno durante a sua formação acadêmica a fatores como as características do ambiente institucional, características e experiências do estudante e a relação entre professor e aluno. Para Murray (2006), esses modelos explicam não apenas a maneira como os estudantes se beneficiam da sua formação, mas também como as IES estão preparadas para fornecer esses benefícios. Ainda segundo a autora, o modelo mais citado nessa área é o EAR de Astin (1993).

Os estudos de Astin (1993) contribuem para o desenvolvimento da área da educação superior. Enfatizam a compreensão de como ocorrem as mudanças e o desenvolvimento dos alunos durante o processo de formação e o que pode ser feito para incrementar esse desenvolvimento (Schleich, 2006). O modelo EAR, segundo Ahmad et al. (2012), é aplicável em quase qualquer campo das ciências sociais e tem sido utilizado em diversos estudos. Esse modelo tem como premissa que as avaliações educacionais não são completas, a menos que incluam informações sobre as entradas, o ambiente educativo e os resultados dos alunos (Astin, 1993).

Os fatores que compõem o modelo são os conceitos fundamentais do modelo teórico do envolvimento do aluno de Astin (1984), o qual descreve a importância do envolvimento dos alunos na universidade. As (i) entradas, são os dados demográficos, a formação e quaisquer experiências anteriores do aluno; (ii) o ambiente é responsável por todas as experiências vividas pelo aluno durante o curso e (iii) os resultados cobrem características, conhecimentos, atitudes, crenças e valores que permanecem depois que o aluno se forma.

Nessa direção, o modelo de Astin é considerado apropriado para explicar o compromisso profissional, uma vez que integra elementos importantes dentro do ensino de Contabilidade. As entradas se referem às características com as quais os alunos entram no curso de graduação em Ciências Contábeis, e influenciam diretamente o ambiente e os resultados. O ambiente é relativo às experiências dos alunos durante o curso. Os resultados são os conhecimentos, as realizações acadêmicas, os valores e os comportamentos adquiridos durante a formação (Ahmad et al., 2012).

Ademais, esse modelo evidencia a necessidade de ter uma compreensão das qualidades dos alunos e as características que apresentam na sua entrada em uma instituição de ensino, o contexto dos ambientes educacionais com os quais entram em contato e a medida da evolução dos conhecimentos e valores adquiridos no decorrer do período acadêmico. As variáveis de entrada dos alunos são as características com as quais eles iniciam a graduação (Astin 1993), e uma delas é a motivação que determina a forma como os alunos trabalham em relação aos seus objetivos (Ahmad et al., 2012). Para Santos (2013), o compromisso com a 
formação universitária é dependente da motivação individual e das habilidades acadêmicas do aluno.

Nesse contexto, para saber como a motivação age sobre o compromisso profissional é necessário identificar quais fatores estão diretamente associados a ela e se são de origem externa ou interna ao indivíduo. No modelo de Astin (1993), a motivação tem relação direta com o ambiente, o que é corroborado por Murray (2006), quando afirma que a motivação é um subproduto da interação com o meio ambiente. Portanto, com relação ao construto motivação, propõe-se as seguintes hipóteses para esta pesquisa:

$\mathbf{H}_{1}$ : A motivação dos alunos tem relação positiva com a percepção do ambiente.

$\mathbf{H}_{2}$ : A motivação dos alunos tem relação positiva com o compromisso profissional.

Da mesma forma que a motivação, a percepção de ambiente é uma variável que afeta indiretamente o compromisso profissional. Para Murray (2006), uma vez que as IES são responsáveis pela criação de um ambiente propício ao aprendizado, a contrapartida dos alunos é a responsabilidade pelo investimento de tempo e de esforço, visando à sua própria educação. Santos (2013) pontua que o envolvimento do aluno, entendido na sua interação e esforço próprio com os sistemas sociais e acadêmicos da universidade, ocorre com o tempo. Nessa mesma linha, Schleich (2006) evidenciou a importância do papel do aluno na troca de ideias sobre o ambiente institucional, as quais representam mudanças que dependem do envolvimento dos estudantes com os recursos oferecidos pela instituição. Com base nesse contexto, elabora-se a terceira hipótese de pesquisa:

$\mathbf{H}_{3}$ : $\mathrm{O}$ ambiente percebido tem relação positiva com o envolvimento do aluno.

O construto envolvimento é o ponto central do modelo de Astin (1984). Em conformidade com 0 autor, os estudantes mais envolvidos dedicam-se com afinco na realização de seus estudos. Empregam parcela considerável de tempo no contexto universitário, participam ativamente de tarefas estudantis e interagem com funcionários e docentes da instituição acadêmica a que pertencem. Assim, o construto de envolvimento do aluno em certos aspectos se assemelha a uma construção mais comum em psicologia: a motivação (Astin, 1984). Portanto, estabelece-se as seguintes hipóteses de pesquisa:

$\mathbf{H}_{4}$ : Motivação do aluno tem relação positiva com o envolvimento do aluno para com a profissão

$\mathbf{H}_{5}$ : $\mathrm{O}$ envolvimento do aluno atua como variável mediadora na influência da motivação sobre o compromisso profissional.

Diante dessas considerações, o modelo teórico integra quatro elementos relevantes do modelo do envolvimento de Astin (1993), a saber: a motivação do aluno, percepção de ambiente, o envolvimento e o compromisso profissional, conforme apresentado na Figura 1.

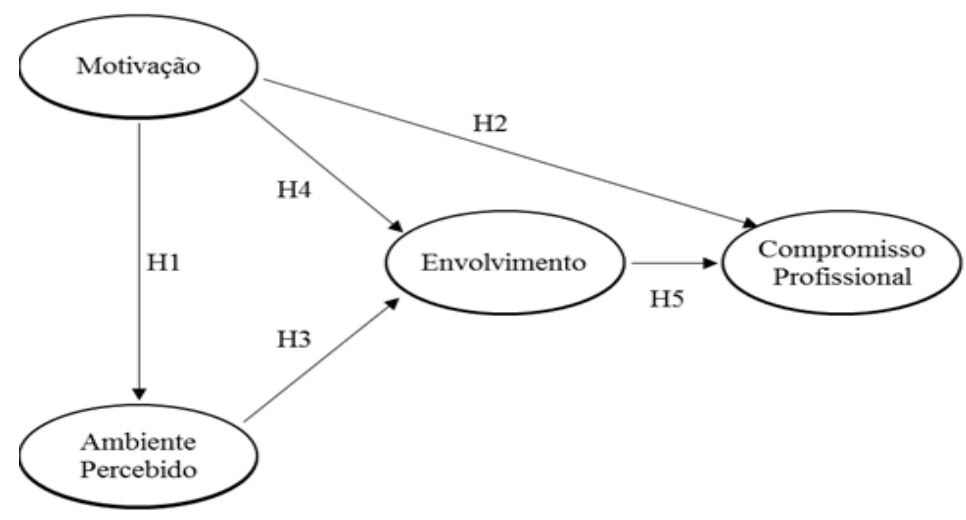

Figura 1. Modelo teórico e hipóteses da pesquisa

Fonte: Ahmad, Z., Anantharaman, R. N., \& Ismail, H. (2012). Student's motivation, perceived environment and professional commitment: An application of Astin's College Impact Model. Accounting Education, 21(2), 187-208.

O próximo tópico apresenta a metodologia adotada para os testes das hipóteses levantadas nesta pesquisa. 


\section{METODOLOGIA}

A população investigada neste estudo é composta por 165 alunos regularmente matriculados nos cursos de graduação em Ciências Contábeis e concluintes no ano de 2016, em duas instituições federais de ensino superior no estado do Paraná. Para calcular o número mínimo da amostra foi utilizado o software $G^{\star} P o w e r$, alinhado aos parâmetros sugeridos por Ringle, Silva e Bido (2014), para uso em modelagem de equações estruturais. O software G*Power possibilita o cálculo da amostra mínima a priori, com base na variável latente com maior número de preditores (Ringle et al., 2014). Para este estudo, considerados os construtos presentes, a amostra mínima calculada foi de 63 respondentes. A amostra final foi composta de 71 alunos.

O instrumento de pesquisa utilizado para coletar os dados neste estudo foi traduzido e adaptado de Ahmad et al. (2012). O questionário original é composto de 69 assertivas, das quais cinco foram excluídas por não serem aplicáveis ao contexto brasileiro. Assim, a versão final foi composta de 64 assertivas em escala do tipo Likert de sete pontos. O processo de Back-translation (Cooper \& Schindler, 2003) foi utilizado na preparação do instrumento aplicado aos potenciais respondentes. Após a tradução e adaptação, foi realizado um pré-teste com seis alunos de um programa de pós-graduação stricto-sensu e com quatro professores de uma universidade federal, com a finalidade de verificar a compreensibilidade do conteúdo e realizar os possíveis ajustes. A Tabela 1 resume a composição do questionário, por construtos, variáveis e respectivas quantidades de assertivas.

Tabela 1

\section{Construtos e variáveis da pesquisa}

\begin{tabular}{l|l|c}
\multicolumn{1}{c|}{ Construtos } & \multicolumn{1}{c}{ Variáveis } & \multicolumn{1}{c}{ Assertivas } \\
\hline Motivação (MTV) & $\begin{array}{l}\text { - Motivação Externa (MTVE); } \\
\text { - Motivação Interna (MTVI); }\end{array}$ & $\begin{array}{c}1 \text { a } 9 \\
\text { Bloco A }\end{array}$ \\
\hline Percepção de Ambiente (P.A.) & $\begin{array}{l}\text { - Alunos (PA.ALU); } \\
\text { - Prédio (PA.PRD); } \\
\text { - Professor (PA.Prof); } \\
\text { - Universidade (PA.UNV) } \\
\text { Envolvimento do aluno (ENV) }\end{array}$ & $\begin{array}{c}1 \text { a } 20 \\
\text { Bloco B }\end{array}$ \\
\hline Comprometimento Profissional (CPROF) com professores (ENV.I); & - Comprometimento Profissional (CPROF) & $\begin{array}{c}1 \text { a } 20 \\
\text { Bloco C }\end{array}$ \\
\hline Bloco D 15
\end{tabular}

Fonte: Adaptado de Ahmad, Z., Anantharaman, R. N., \& Ismail, H. (2012). Student's motivation, perceived environment and professional commitment: An application of Astin's College Impact Model. Accounting Education, 21(2), 187-208.

Para coleta dos dados, o instrumento de pesquisa foi aplicado presencialmente aos alunos por um dos autores, no mês de abril de 2016, nas duas instituições de ensino federal, ambas no estado do Paraná.

Os dados coletados foram inicialmente tratados com técnicas de estatística descritiva. Posteriormente, foi utilizada a técnica de Modelagem de Equações Estruturais (Structural Equations Modeling (SEM)) e processados com o software SmartPLS. A SEM é uma técnica de estimação de regressão linear baseada na decomposição de matrizes de variáveis e de covariáveis (Bido, Silva, Souza, \& Godoy, 2010). A SEM permite testar um conjunto de variáveis e investigar o nível de explicação das variáveis independentes frente às variáveis dependentes (aspectos de regressão múltipla), e indicar qual variável exógena é mais importante (Klem, 2006). Neste estudo, a opção pelo modelo Partial Least Squares (PLS) devese ao tamanho da amostra $(n=71)$, já que é apropriado quando se trabalha com amostras pequenas (Hair, Black, Babin, Anderson, \& Tatham, 2009).

\section{RESULTADOS E DISCUSSÃO}

Nesta seção serão apresentados, em um primeiro momento, o perfil dos respondentes, 
a análise descritiva dos dados, validade e confiabilidade do modelo. Na sequência, o modelo conceitual e o teste estatístico, e, por fim, a análise e discussão dos resultados.

\subsection{Perfil dos respondentes}

$\mathrm{Na}$ Tabela 2 estão detalhadas as características da amostra, ou seja, o perfil demográfico quanto a gênero, idade, instituição e estado civil dos 71 respondentes da pesquisa.

Tabela 2

Perfil dos respondentes

\begin{tabular}{lcc|lcc}
\multicolumn{1}{c}{ Gênero } & $\mathbf{N}$ & $\%$ & \multicolumn{1}{c}{ Idade } & N & $\%$ \\
\hline Feminino & 38 & $54 \%$ & De 1970 a 1979 & 1 & $1 \%$ \\
Masculino & 33 & $46 \%$ & De 1980a 1989 & 15 & $21 \%$ \\
& & & De 1990 a 1995 & 55 & $77 \%$ \\
\hline \multicolumn{1}{c}{ Instituição } & $\mathbf{N}$ & $\%$ & \multicolumn{1}{c}{ Estado civil } & $\mathbf{N}$ & $\%$ \\
\hline UFPR & 45 & $63 \%$ & Casado (a) & 14 & $20 \%$ \\
UTFPR & 26 & $37 \%$ & Divorciado (a) & 2 & $3 \%$ \\
& & & Solteiro (a) & 55 & $77 \%$ \\
\hline
\end{tabular}

Fonte: dados da pesquisa (2016).

Conforme Tabela 2, dos 71 respondentes da pesquisa, 54\% são do gênero feminino, com preponderância de idade no intervalo 21 e 26 anos. Da amostra total, 45 alunos estudam na Universidade Federal do Paraná (UFPR), localizada em Curitiba, e 26 na Universidade Tecnológica Federal do Paraná, situada em Pato Branco. Quanto ao estado civil, 55 respondentes declaram-se solteiros (as). Dos 71 alunos respondentes e regularmente matriculados como concluintes do ano de 2016, 18 deles estão em seu segundo curso de ensino superior. Entre os cursos concluídos destacam-se: Design de Interiores, Turismo, Administração, Zootecnia, Letras, Logística, Direito, Secretariado, Matemática, Engenharia Ambiental e Física. Não foi investigado o motivo pelo qual os alunos estão frequentando um segundo curso, em específico, o de graduação em Ciências Contábeis.

\subsection{Modelo estrutural e hipóteses de pesquisa}

A qualidade do modelo foi avaliada por meio da confiabilidade composta (CC), Alfa de Cronbach, validade convergente e validade discriminante. A confiabilidade é considerada apropriada quando as cargas fatoriais apresentam um Alfa de Cronbach e uma CC de pelo menos 0,70 (Hair et al., 2009). A validade convergente, em conformidade com os argumentos de Hair et al. (2009), refere-se à variância média extraída (Average Variance Extracted (AVE)), que demonstra a variância compartilhada entre os indicadores de cada variável latente ou construto do modelo.

A análise da validade discriminante foi feita comparando-se a raiz quadrada da AVE com as correlações entre as variáveis latentes (Fornell \& Larcker, 1981). Esse indicador é considerado satisfatório quando o coeficiente obtido dos construtos latentes, manifestado nas suas variáveis, excede a 0,50 (Fornell \& Larcker, 1981).

Finalizados os referidos testes, foi identificada a necessidade de exclusão de alguns indicadores: (i) motivação externa (MTVE4, MTVE 5, MTVE 6); (ii) percepção do ambiente alunos (PA.ALU2), universidade (PA.UNV4); (iii) envolvimento-interação com professores (ENV.I1, ENV.I10, ENV.I13, ENV.I14), esforço próprio (ENV.P1, ENV.P6) e (iv) comprometimento profissional (CPROF2, CPROF 3, CPROF 4, CPROF 6, CPROF 8). A Tabela 3 detalha os resultados da AVE, CC, $\mathrm{R}^{2}$ e Alfa de Cronbach. 
Tabela 3

Confiabilidade interna dos construtos e validade convergente

\begin{tabular}{l|c|c|c|c}
\multicolumn{1}{c|}{ Variáveis } & $\boldsymbol{A V E}$ & Confiabilidade Composta & $\boldsymbol{R}^{2}$ & Alfa de Cronbach \\
\hline PA.ALU & 0,7016 & 0,9037 & 0,112 & 0,8574 \\
\hline PA.Prof & 0,6649 & 0,9082 & 0,253 & 0,8744 \\
\hline ENV.I & 0,5469 & 0,8252 & 0,334 & 0,7303 \\
\hline ENV.P & 0,3811 & 0,8578 & 0,354 & 0,8177 \\
\hline MTVE & 0,6601 & 0,8535 & - & 0,7536 \\
\hline MTVI & 0,6685 & 0,8580 & - & 0,7521 \\
\hline PA.PDR & 0,7752 & 0,9451 & 0,208 & 0,9274 \\
\hline CPROF & 0,5645 & 0,9273 & 0,433 & 0,9112 \\
\hline PA.UNV & 0,6231 & 0,8686 & 0,179 & 0,7996 \\
\hline
\end{tabular}

Nota. Alunos (PA.ALU); Professor (PA.Prof); Interação com professores (ENV.I); Esforço próprio (ENV.P); Motivação Externa (MTVE); Motivação Interna (MTVI); Prédio (PA.PRD); Comprometimento Profissional (CPROF); Universidade (PA.UNV).

Fonte: dados da pesquisa (2016).

Os coeficientes de AVE detalhados na Tabela 3 atendem aos parâmetros indicados na literatura, com exceção da dimensão envolvimento de esforço próprio. Mesmo após as exclusões de dois indicadores, identificados com baixas cargas fatoriais (ENV.P1, ENV.P6), não se conseguiu atender por completo à validade convergente. Contudo, devido ao fato dessa dimensão compor o construto envolvimento componente do modelo de Astin, a opção foi pela sua permanência. Em relação à confiabilidade do modelo, os valores para a confiabilidade composta e para o Alfa de Cronbach são superiores a 0,70, o mínimo recomendado. A Tabela 4 detalha os resultados referentes à validade discriminante.

Tabela 4

Validade discriminante

\begin{tabular}{l|c|c|c|c|c|c|c|c|c} 
Variáveis & PA.ALU & PA.Prof & ENV.I & ENV.P & MTVE & MTVI & PA.PDR & CPROF & PA.UNV \\
\hline PA.ALU & $\mathbf{0 , 8 3 7 6}$ & & & & & & & & \\
\hline PA.Prof & 0,5810 & $\mathbf{0 , 8 1 5 4}$ & & & & & & & \\
\hline ENV.I & 0,4281 & 0,4961 & $\mathbf{0 , 7 3 9 5}$ & & & & & & \\
\hline ENV.P & 0,3485 & 0,4178 & 0,4967 & $\mathbf{0 , 6 1 7 3}$ & & & & & \\
\hline MTVE & 0,1773 & 0,4419 & 0,1406 & 0,4722 & $\mathbf{0 , 8 1 2 5}$ & & & & \\
\hline MTVI & 0,3235 & 0,3592 & 0,2906 & 0,3272 & 0,2910 & $\mathbf{0 , 8 1 7 6}$ & & & \\
\hline PA.PDR & 0,5974 & 0,6367 & 0,2987 & 0,2095 & 0,2583 & 0,4342 & $\mathbf{0 , 8 8 0 5}$ & & \\
\hline CPROF & 0,4763 & 0,5504 & 0,4048 & 0,4181 & 0,4622 & 0,5143 & 0,5355 & $\mathbf{0 , 7 5 1 3}$ & \\
\hline PA.UNV & 0,6518 & 0,7652 & 0,5033 & 0,3855 & 0,3115 & 0,3647 & 0,6812 & 0,4915 & $\mathbf{0 , 7 8 9 4}$ \\
\hline Font:
\end{tabular}

Fonte: dados da pesquisa (2016).

De modo geral, conforme indicado nos resultados evidenciados na Tabela 4, a validade discriminante foi amplamente atendida por meio dos coeficientes da raiz quadrada da AVE (Fornell \& Larcker, 1981).

Com base no modelo Astin (1993) e de Ahmad et al. (2012), foram enunciadas cinco hipóteses, como respostas tentativas à questão de pesquisa. A corroboração ou não dessas hipóteses de pesquisa foram feitas por meio do procedimento de Bootsttrapping. Em cada caminho estrutural do diagrama dos caminhos (path diagram) do modelo, o teste para corroborar ou não a hipótese de pesquisa foi verificar se os valores dos $t$-valor são superiores a 1,96 para um p-valor $<0,01$ e p-valor $<0,05$, conforme recomendado por Hair et al. (2009). A Tabela 5 detalha a análise das relações estabelecidas entre os construtos investigados. 
Tabela 5

Painel das relações entre os construtos investigados

\begin{tabular}{|c|c|c|c|c|c|c|}
\hline \multicolumn{7}{|c|}{ PAINEL A: sem mediação } \\
\hline Hipótese & Construtos & Relação estrutural & Beta & t-valor & p-valor & Sig. \\
\hline \multirow{8}{*}{$\mathrm{H} 1$} & \multirow{8}{*}{ MTV-> PA } & MTVE-> PA.ALU & 0,0908 & 0,7685 & 0,442 & n.s \\
\hline & & MTVE-> PA.Prof & 0,3686 & 3,3526 & $0,001 *$ & sig \\
\hline & & MTVE-> PA.PRD & 0,1442 & 1,091 & 0,276 & n.s \\
\hline & & MTVE-> PA.UNV & 0,2244 & 1,9572 & 0,051 & n.s \\
\hline & & MTVI-> PA.ALU & 0,2971 & 2,6894 & $0,007^{*}$ & $\operatorname{sig}$ \\
\hline & & MTVI-> PA.Prof & 0,2519 & 2,5117 & $0,012^{* *}$ & sig \\
\hline & & MTVI-> PA.PRD & 0,3922 & 3,7827 & 0,000 * & sig \\
\hline & & MTVI-> PA.UNV & 0,2994 & 2,745 & $0,006^{*}$ & sig \\
\hline \multirow{2}{*}{$\mathrm{H} 2$} & \multirow{2}{*}{ MTV-> CPROF } & MTVE-> CPROF & 0,3102 & 2,769 & $0,006^{*}$ & sig \\
\hline & & MTVI-> CPROF & 0,3405 & 3,5858 & 0,000 * & sig \\
\hline \multirow{8}{*}{ H3 } & \multirow{8}{*}{$\mathrm{PA}->\mathrm{ENV}$} & PA.ALU -> ENV.P & 0,1653 & 0,912 & 0,362 & n.s \\
\hline & & PA.ALU -> ENV.I & 0,215 & 1,2133 & 0,225 & n.s \\
\hline & & PA.Prof -> ENV.P & 0,3242 & 2,0192 & $0,044^{* *}$ & sig \\
\hline & & PA.Prof. -> ENV.I & 0,1269 & 0,6732 & 0,501 & n.s \\
\hline & & PA.PRD -> ENV.P & $-0,243$ & 1,375 & 0,169 & n.s \\
\hline & & PA.PRD -> ENV.I & $-0,277$ & 1,5322 & 0,126 & n.s \\
\hline & & PA.UNV -> ENV.P & 0,2901 & 1,58 & 0,114 & n.s \\
\hline & & PA.UNV -> ENV.I & 0,1664 & 0,7686 & 0,442 & n.s \\
\hline \multirow{4}{*}{$\mathrm{H} 4$} & \multirow{4}{*}{ MTV->ENV } & MTVE-> ENV.P & $-0,103$ & 0,8897 & 0,374 & n.s \\
\hline & & MTVE-> ENV.I & 0,3481 & 2,3637 & 0,018 ** & sig \\
\hline & & MTVI-> ENV.I. & 0,1703 & 1,0809 & 0,280 & n.s \\
\hline & & MTVI-> ENV.P & 0,1503 & 1,2266 & 0,220 & n.s \\
\hline \multicolumn{7}{|c|}{ PAINEL B : com mediação } \\
\hline Hipótese & Construtos & Relação estrutural & $\begin{array}{c}\text { Ef. } \\
\text { Direto }\end{array}$ & $\begin{array}{c}\text { Ef. } \\
\text { Indireto }\end{array}$ & Ef. Total & Sig \\
\hline \multirow{4}{*}{ H5 } & \multirow{4}{*}{ MTV->ENV-> CPROF } & MTVE-> ENV.I -> CPROF & 0,3102 & 0,0138 & 0,3240 & n.s \\
\hline & & MTVE-> ENV.P -> CPROF & 0,3102 & $-0,0250$ & 0,2852 & n.s \\
\hline & & MTVI-> ENV.I -> CPROF & 0,3405 & 0,0068 & 0,3473 & n.s \\
\hline & & MTVI-> ENV.P.-> CPROF & 0,3405 & 0,0364 & 0,3769 & n.s \\
\hline
\end{tabular}

Nota. n.s.: não significativo e, sig.: significativo.

Fonte: dados da pesquisa (2016).

Os dados dos testes detalhados na Tabela 5 evidenciam as relações entre as dimensões de todos os construtos, individualizadas para melhor visualização. Os resultados das análises do teste de hipóteses da pesquisa estão de acordo com os procedimentos utilizados no estudo de Ahmad et al. (2012).

A H1 advoga que a motivação dos alunos é positivamente associada à percepção do ambiente. Diferentemente do achado por Ahmad et al. (2012), não foi corroborada. O construto motivação é composto pelas dimensões (i) motivação externa e (ii) motivação interna. No entanto, na análise de validação do modelo estrutural, três indicadores foram excluídos por não atenderem aos valores recomendados (discriminante e convergente). Das relações finais obtidas, apenas as relações MTVE-> PA.ALU; MTVE-> PA.PRD, e MTVE. -> PA.UNV não foram estatisticamente significativas. Portanto, os resultados evidenciam que a dimensão motivação interna é a mais forte, sugerindo que os respondentes possuem lócus de controle interno preponderante.

A H2 descreve que a motivação dos alunos é positivamente associada ao compromisso profissional, o que foi corroborado, a um nível de confiança de $99 \%$, para as dimensões (MTVE $\rightarrow$ CPROF $\operatorname{com} \beta=0,3102, t=2,769, p<0,01$ e, MTVI->CPROF $\operatorname{com} \beta=0,3405, t=3,5858, p$ $<0,01$ ), aderente com os achados do estudo de Ahmad et al. (2012). Esse achado sugere que os respondentes demonstram comprometimento profissional mediante fatores motivacionais internos e externos.

A H3, a qual descreve que o ambiente percebido tem relação positiva com 0 
envolvimento do aluno, não foi corroborada, divergindo dos achados de Ahmad et al. (2012). A única exceção, positivamente significativa, é a relação entre as dimensões PA.Prof $\rightarrow$ ENV.P $(\beta$ $=0,3242, t=2,0192, p<0,05)$. Esse resultado evidencia que os professores do ambiente institucional afetam o esforço próprio dos estudantes. Portanto, sugere que os professores exercem grande influência de um maior comprometimento próprio para com a formação e, consequentemente, com a profissão escolhida pelo aluno.

A H4, de que a motivação do aluno é positivamente associada ao seu envolvimento com o curso de Ciências Contábeis e com a profissão contábil, não foi confirmada. A motivação interna não se caracteriza como uma dimensão que afeta o envolvimento dos alunos para com os professores e para com seu esforço pessoal. Outro detalhe é que a motivação externa não afeta o envolvimento dos alunos com o esforço próprio, ou seja, que influências e opiniões externas não impactam significativamente em um maior esforço do estudante.

Por fim, a H5 buscava testar se o envolvimento do aluno atua como variável mediadora na influência da motivação sobre o compromisso profissional, e, diferentemente do achado do estudo de Ahmad et al. (2012), não foi corroborada. Esse resultado sugere que a motivação, nas dimensões externa e interna, não afeta o comprometimento profissional, por meio do envolvimento de comprometimento pessoal e também por meio do envolvimento com os professores.

Portanto, os achados desta investigação, realizada em um ambiente de ensino de duas instituições federais de ensino superior do estado do Paraná, divergem, em parte, dos do estudo de Ahmad et al. (2012). Em síntese, os resultados sugerem que as percepções de motivação e de ambiente não impactam de forma estatisticamente significativa o envolvimento, porém a motivação afeta de forma positiva e significativa o comprometimento profissional. Revela que um aluno motivado com a profissão será um bom profissional.

\section{CONCLUSÕES}

Este estudo investigou os fatores de socialização antecipatória de estudantes universitários de Contabilidade de duas universidades federais, localizadas no estado do Paraná, com base no estudo de Astin. Para coleta dos dados, utilizou-se um questionário adaptado do estudo de Ahmad et al. (2012), aplicado a 71 alunos concluintes da graduação no ano de 2016. Para o tratamento dos dados, utilizou-se técnicas estatísticas de análise descritiva e de modelagem de equações estruturais via PLS.

Os resultados da pesquisa referentes à analise descritiva apontam que a amostra foi composta por $54 \%$ de mulheres, com respondentes de faixa etária predominantemente entre 21 e 26 anos. Para analisar as hipóteses, utilizou-se a modelagem de equações estruturais. Conforme os resultados obtidos, infere-se que a dimensão motivação interna é a mais forte, quando comparada com motivação originada por fatores externos. Evidencia que os alunos questionados possuem lócus de controle interno preponderante. Com isso, depreende-se que o construto motivação de forma completa só afeta de forma estatisticamente significativa a variável compromisso profissional.

A pesquisa teve como objetivo investigar os fatores que durante o curso de Ciências Contábeis influenciam a construção do compromisso profissional dos alunos com a profissão contábil. $\mathrm{Na}$ análise dos resultados, a variável motivação, composta pelos fatores internos e externos, influencia positivamente o compromisso profissional dos alunos de Ciências Contábeis quanto à profissão contábil. Ademais, na análise da variável motivação, a dimensão interna foi fortemente relacionada à percepção do ambiente. Corroborando tal resultado, Murray (2006) afirma que a motivação é um subproduto da interação com o ambiente e, por isso, a instituição tem grande responsabilidade para essa melhoria.

Ainda, em relação ao ambiente percebido, constatou-se que os professores exercem grande influência no comprometimento profissional do aluno e, consequentemente, em sua formação profissional. Consoante este resultado, Krug e Krug (2008) advogam que o professor pode influenciar os alunos, quer como pessoas, quer como profissionais, ou seja, quanto mais presentes são os professores na formação, melhor o comprometimento e a percepção do ambiente $o$ aluno terá. 
Um achado interessante da análise aponta que a motivação não afeta o compromisso profissional por meio do envolvimento do aluno. Para Murray (2006), uma vez que as IES são responsáveis pela criação de um ambiente propício ao aprendizado, a contrapartida dos alunos é a responsabilidade pelo investimento de tempo e de esforço visando sua própria educação. Nesse sentido, vale ressaltar aos alunos que, quando da escolha do curso superior, e consequentemente da futura profissão, devem se respaldar das mais completas informações para ter certeza da decisão. Com isso, mais assertiva será a decisão em relação ao curso e à profissão e, consequentemente, maior será o comprometimento do indivíduo.

A pesquisa apresenta como limitação o fato de terem sido investigados alunos de apenas duas IES públicas, o que faz com que os resultados não possam ser generalizados. Outro fator limitante é que somente foram investigados alunos do último ano do curso de Ciências Contábeis em ambas instituições. Os achados instigam a realização de estudos em outras instituições, inclusive as privadas, que podem trazer resultados diferentes, principalmente no que tange ao construto "ambiente", devido às especificidades dessas IES em relação à sua estrutura organizacional. Também se recomenda que a investigação se estenda aos outros períodos da graduação, não se limitando aos alunos concluintes do curso, visando capturar também as percepções dos demais em relação aos construtos do modelo.

\section{REFERÊNCIAS}

Ahmad, Z., Anantharaman, R. N., \& Ismail, H. (2012). Student's motivation, perceived environment and professional commitment: An application of Astin's College Impact Model. Accounting Education, 21(2), 187-208.

Ardts, J., Janse, P., \& Van der Velde, M. (2001). The breaking in of new employees: Effectiveness of socialization tactics and personnel instruments. The Journal of Management Development, 20(2), 159-167.

Astin, A. W. (1993). Assessment for excellence: The philosophy and practice of assessment and evaluation in higher education. The Journal of Higher Education, 63(6), 717-720.

Astin, A. W. (1984). Student involvement: A developmental theory for higher education. Journal of College Student Personnel, 25(4), 297-308.

Baccaro, T. A. (2007). Influência da escolha vocacional no processo de socialização profissional de estudantes de enfermagem. Dissertação de Mestrado, Faculdade de Economia e Administração e Contabilidade de Ribeiro Preto, Ribeirão Preto, São Paulo, Brasil.

Baccaro, T. A., \& Shinyashiki, G. T. (2011). Consistência da escolha vocacional e socialização profissional de estudantes de enfermagem. Revista Brasileira de Orientação Profissional, 12(1), 73-82.

Barros, I. M. D. C. (2011). Contributo para a compreensão do processo de (re) construção da identidade profissional no contexto da formação inicial: estudo em estudantes estagiários de educação física. Dissertação de Mestrado, Universidade do Porto, Faculdade de Desporto, Porto, Portugal.

Bastos, V. B., A., \& Andrade, J. E. B. (2002). Comprometimento com o trabalho: padrões em diferentes contextos organizacionais. Revista de Administração de Empresas, 42(2), 111. 
Bido, D., Silva, D., Souza, C. A., \& Godoy, A. S. (2010). Mensuração com indicadores formativos nas pesquisas em administração de empresas: como lidar com a multicolinearidade entre eles? Administração: Ensino e Pesquisa, 11(2), 245-269.

Borges, E., \& Medeiros, C. (2007). Comprometimento e ética profissional: um estudo de suas relações junto aos contabilistas. Revista de Contabilidade e Finanças, 18(44), 60-71.

Cooper, D. R. \& Schindler, P. S. (2003). Métodos de Pesquisa em Administração (7a ed.). Porto Alegre: Bookman.

Dubar, C. (2003). A socialização: construção das identidades sociais e profissionais. São Paulo: Martins Fontes.

Elias, R. Z. (2006). The impact of professional commitment and anticipatory socialization on accounting students' ethical orientation. Journal of Business Ethics, 68(1), 83-90.

Elias, R. Z. (2007). The relationship between auditing student's anticipatory socialization and their professional commitment. Academy of Educational Leadership Journal, 11(1), 81.

Elias, R. Z. (2008). Auditing student's professional commitment, anticipatory socialization, and their relationship to whistleblowing, Managerial Auditing Journal, 23(3), 283-294.

Everett, J., \& Tremblay, M. S. (2014). Ethics and internal audit: Moral will and moral skill in a heteronomous field. Critical Perspectives on Accounting, 25(3), 181-196.

Farag, M. S., \& Elias, R. Z. (2016). The relationship between accounting students personality, professional skepticism and anticipatory socialization. Accounting Education, 25(2), 124138.

Fornell, C. \& Larcker, D. F. (1981). Structural equation models with unobservable variables and measurement error: Algebra and statistics. Journal of marketing research, 382-388.

Hair, J. F., Jr., Black, W. C., Babin, B. J., Anderson, R. E., \& Tatham, R. L. (2009). Análise multivariada de dados (6a ed.). Porto Alegre: Bookman.

Klem, L. (2006). Structural equation modeling. In Grimm, L. G. \& Yarnold, P. R. (Eds.). Reading and understanding more multivariate statistics. Washington: American Psychological Association.

Krug, R. R., \& Krug, H. N. (2008). As gratificações e frustrações da docência em Educação Física Escolar para os acadêmicos do CEFD/UFSM em situações de estágio. Revista Digital Lecturas: Educación Física y Deportes, 13(125), 1-10.

Low, M., Davey, H., \& Hooper, K. (2008). Accounting scandals, ethical dilemmas and educational challenges. Critical perspectives on Accounting, 19(2), 222-254.

Mahdavikhou, M., \& Khotanlou, M. (2012). New approach to teaching of ethics in accounting introducing Islamic ethics into accounting education. Procedia-Social and Behavioral Sciences, 46, 1318-1322.

Mastracchio, N. J. (2005). Teaching CPAs about serving the public interest. The CPA Journal 76, 6.

Murray, M. C. (2006). Reframing Responsibility for Academic Success: A Causal Model Measuring the Impact of Student Attributes in the First Year of College. Ph.D. Dissertation, Faculty of the Graduate School of the Maryland, College Park, USA. 
Pascarella, E. T. \& Terenzini, P. T. (2005). How college affects students: $A$ third decade of research. San Francisco: Jossey-Bass Publishers.

Pitney, W. A. (2002). The professional socialization of certified athletic trainers in high school settings: A grounded theory investigation. Journal of Athletic Training, 37(3), 286-292.

Ringle, C. M., Silva, D., \& Bido, D. (2014). Modelagem de equações estruturais com utilização do SmartPLS. Revista Brasileira de Marketing, 13(2) 56-73.

Santos, P. V. S. (2013). Adaptação à universidade dos estudantes cotistas e não cotistas: relação entre vivência acadêmica e intenção de evasão. Dissertação Mestrado, Universidade Federal da Bahia, Salvador, Bahia, Brasil.

Setyadi, E. J. (2008). The relationship between professional commitment and socialization to repress the orientation of the Ethics of Accounting Students. Diponegoro University, Jawa Tengah, Indonésia.

Schleich, A. L. R. (2006). Integração a educação superior e satisfação acadêmica de estudantes ingressantes e concluintes: um estudo sobre relações. Dissertação de Mestrado de Educação, Universidade Estadual de Campinas, Campinas, São Paulo, Brasil.

Saat, M. M., Porter, S., \& Woodbine, G. (2012). A longitudinal study of accounting students' ethical judgment making ability. Accounting Education, 21(3), 215-229.

Shinyashiki, G. T., Mendes, I. A. C., Trevizan, M. A., \& Day, R. A. (2006). Professional socialization: students becoming nurses. Revista Latino-Americana de Enfermagem, 14(4), 601-607.

Smith, L. M. (2003). A fresh look at accounting ethics (or Dr. Smith goes to Washington). Accounting Horizons, 17(1), 47-49.

Waugaman, W. R., \& Lohrer, D. J. (2000). From nurse to nurse anesthetist: The influence of age and gender on professional socialization and career commitment of advanced practice nurses. Journal of Professional Nursing, 16(1), 47-5.

Weidman, J. C., Twale, D. J., \& Stein, E. L. (2001). Socialization of Graduate and Professional Students in Higher Education: A Perilous Passage? ASHE-ERIC Higher Education Report, Volume 28, Number 3. Jossey-Bass Higher and Adult Education Series. JosseyBass, Publishers, Inc., 350 Sansome Street, San Francisco, CA 94104-1342. 